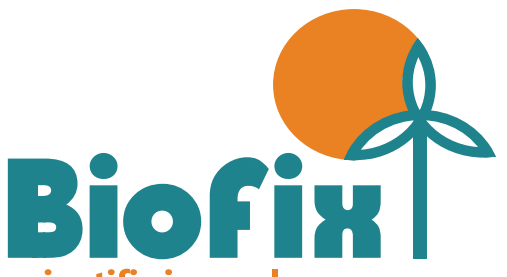

scientific journal

v. 2 n. Especial I MECFOR 2017

\title{
PRODUTIVIDADE DO PROCESSAMENTO MECANIZADO DA MADEIRA DE PINUS NOS SISTEMAS FULL TREE E CUT TO LENGTH EM DIFERENTES VOLUMES INDIVIDUAIS
}

\author{
PRODUCTIVITY OF MECHANIZED PINEWOOD PROCESSING \\ IN FULL TREE AND CUT TO LENGTH SYSTEMS IN \\ DIFFERENT INDIVIDUAL VOLUMES
}

Recebido em 05/11/2017

Aceito em 14/11/2017

Publicado em 22/11/2017

DOI: dx.doi.org/10.5380/biofix.v2i0.56344

Antônio José Scorupski ${ }^{1}$ Felipe Martins de Oliveira ${ }^{2,5}$ Carlos Cézar Cavassin Diniz ${ }^{3}$ Eduardo da Silva Lopes ${ }^{4}$

Faculdades FatiFajar, Jaguariaíva, Paraná, Brasil antonio.scorupski@outlook.com ${ }^{1}$ \& eng.oliveirafm@gmail.com ${ }^{2}$

Universidade Federal do Paraná, Curitiba, Paraná, Brasil carlos.diniz@ufpr.br

Universidade Estadual do Centro-Oeste, Irati, Paraná, Brasil eslopes@unicentro.br ${ }^{4}$ \& fmoliveira@unicentro.br ${ }^{5}$

\section{RESUMO}

Este trabalho objetivou analisar o processamento da madeira na colheita mecanizada de Pinus sp. nos sistemas full tree e cut to length, para verificar qual apresenta maior rendimento de acordo com o volume médio individual (VMI) das árvores. O estudo foi realizado nas áreas operacionais de colheita da madeira de uma empresa florestal nos municípios de Jaguariaíva e Sengés, estado do Paraná, em povoamentos de 15 anos de idade em regime de corte raso. A coleta de dados foi realizada durante 12 meses por meio de controles diários de produção, com base no computador de bordo de cada máquina e na separação por classes de VMI, com os dados interpretados por meio de análise gráfica. Os resultados mostraram que houve equilíbrio nas produtividades do processamento em ambos os sistemas na classe de VMI entre 0,21 a $0,25 \mathrm{~m}^{3}$, porém o sistema cut to length demonstrou maior produtividade do processamento nas classes de VMI menores, enquanto o sistema full tree foi superior nas classes de maior VMI, demonstrando a adequabilidade dos dois sistemas para os diferentes volumes dos povoamentos florestais.

PALAVRAS-CHAVE: Colheita florestal, Harvester, Planejamento da colheita.

\section{ABSTRACT}

This work aimed to analyze the wood processing in mechanized Pinus sp. harvesting in both full tree and cut to length systems, to verify which shows the highest efficiency according to the individual average volume (IAV) of the trees. The study was carried out in operational areas of timber harvesting of a forestry company in Jaguariaíva and Sengés, Paraná State, Brazil, in the clear-cut of 15year-old plantations. Data collection was carried out for 12 months by means of daily production controls, based on the onboard computer of each machine and the separation by classes of IAV, with interpretation through graphical analysis. The results showed that there was equilibrium in the productivity of the processing in both systems in the IAV class between 0.21 and $0.25 \mathrm{~m}^{3}$, but cut to length system showed higher processing productivity in the lower IAV classes, while full tree was superior in the classes of higher IAV. Therefore, we demonstrated the suitability of the two systems for different volumes of forest stands.

KEYWORDS: Forestry harvesting, Harvester, Harvest planning. 


\section{INTRODUÇÃO}

A produtividade dos sistemas de colheita florestal é uma das principais variáveis que influenciam na viabilidade da colheita de madeira em florestas plantadas. Segundo Lopes (2001), ela sofre influência de vários fatores técnicos, ambientais, ergonômicos e sociais, os quais impactam diretamente na forma de execução das operações. Apesar do processo contínuo de evolução tecnológica, a colheita da madeira em conjunto com o transporte possui grande importância em termos econômicos dentro da cadeia produtiva da madeira, representando em torno de $50 \%$ ou mais do custo final da madeira posto na indústria (MACHADO, 2014).

Há questionamentos sobre qual o melhor sistema de colheita a ser utilizado para cada situação, em que há necessidade da realização de estudos para analisar operacionalmente os diferentes sistemas de colheita de madeira, visando o melhor entendimento das relações entre as variáveis que explicam esse processo. Com isso, é possível subsidiar o planejamento e a tomada de decisão em um modelo racional para a execução das operações da colheita florestal de forma eficiente e economicamente viável. Por fim, contribui-se para a adequação futura na alocação dos módulos de colheita mecanizada, bem como na escolha ou substituição ideal dos equipamentos.

O processamento da madeira é um dos elementos de maior influência no rendimento das operações. De acordo com Drinko et al. (2015), ele ocupa a maior parte do ciclo operacional do processamento à beira da estrada e do corte com harvester no interior do talhão. Dessa forma, é uma atividade importante para ser avalaida em análises de sistemas de colheita da madeira.

O objetivo deste trabalho foi analisar os dados de processamento da madeira na colheita mecanizada de Pinus sp. nos sistemas full tree e cut to length, visando verificar qual apresenta maior o rendimento de acordo com o volume médio individual das árvores, bem como a futura readequação da colheita.

\section{MATERIAL E MÉTODOS}

O estudo foi realizado na região dos municípios de Jaguariaíva e Sengés, estado do Paraná, Brasil. A área de estudo pertence a uma empresa do segmento de produção de madeira em toras, cuja finalidade é comercializar a produção no final do ciclo aos mercados de madeira serrada, laminação, chapas e celulose.

A área experimental foi composta por plantios de Pinus sp. em duas formas de manejo: utility wood, com um ou dois desbastes programados para comercialização da madeira para serrarias; e pulp wood, com corte final em torno dos 15 anos, com objetivo de fornecimento para indústrias de chapas e papel, sem intervenção de desbastes. Em ambos casos, a colheita foi realizada aos 15 anos, com densidade inicial de 1.666 árvores ha-1.

As máquinas de colheita utilizadas nos dois sistemas foram da mesma marca e modelo, com rodados de esteiras, $111 \mathrm{~kW}$ de potência bruta, peso operacional de $20.970 \mathrm{~kg}$, tanque de combustível com capacidade para 410 litros, acoplada com cabeçote para derrubada, desgalhamento e traçamento com 1,74 m de altura, 1.619 $\mathrm{kg}, 0,71 \mathrm{~m}$ de abertura máxima dos rolos, $90 \mathrm{~cm}$ de sabre e $75 \mathrm{~cm}$ de capacidade máxima de corte (Figura 1).

(A)

(B)
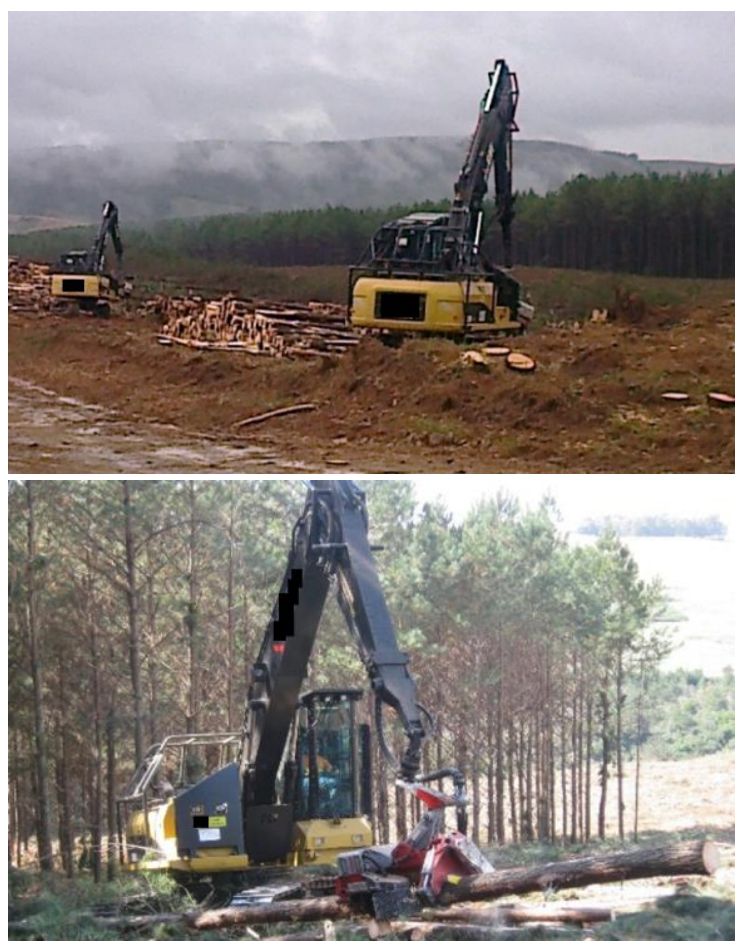

Figura 1. Máquinas utilizadas para o processamento da madeira nos sistemas full tree (A) e cut to length (B).

A coleta de dados foi realizada mediante informações de 12 meses consecutivos de produção, em cada sistema de colheita, no processamento à beira da estrada (sistema full tree) e no interior do talhão (sistema cut to length). Considerou-se cada mês como uma amostra completa e efetuou-se a filtragem dos controles diários de produção, com base no computador de bordo de cada máquina, com as variáveis: horas trabalhadas, número de árvores produzidas e produção de cada um dos turnos considerados.

Com os dados mensais de cada sistema, realizou-se a distribuição nas classes de VMIs, estipuladas de forma 
sistemática em intervalos de $0,05 \mathrm{~m}^{3}$, totalizando 10 classes, até o limite observado de $0,50 \mathrm{~m}^{3}$. Os resultados mensais foram agregados para a somatória total do período, analisados e comparados conforme os objetivos deste trabalho. Por fim, realizou-se a análise gráfica dos resultados obtidos.

\section{RESULTADOS E DISCUSSÃO}

Os dados relativos às produtividades (rendimentos) do processamento da madeira em ambos os sistemas de colheita estudados, por classe de VMI, estão apresentados na Figura 2.

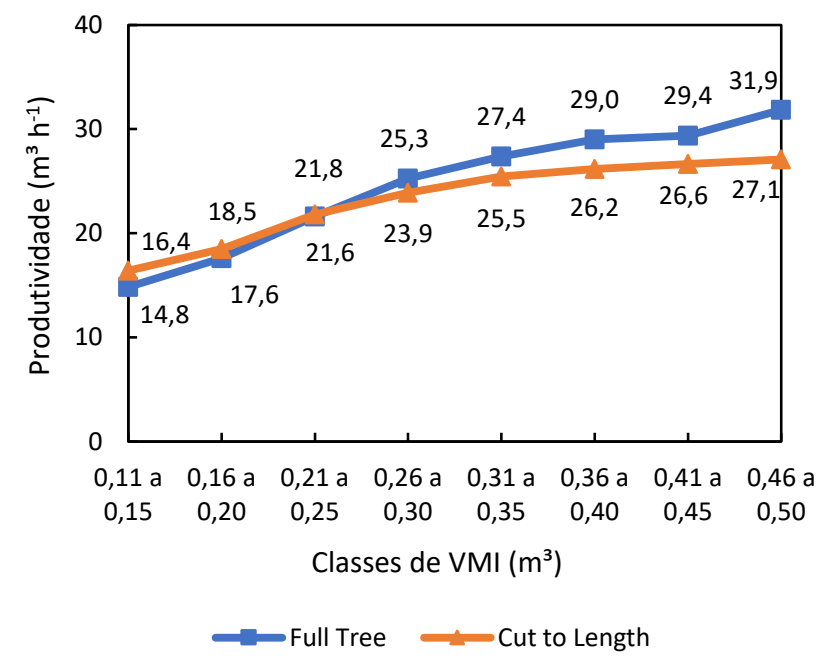

Figura 2. Distribuição dos rendimentos de acordo com os VMIs, considerando somente o processamento.

Observou-se que o equilíbrio de rendimentos no processamento aconteceu na classe de VMI 0,21 a 0,25 $\mathrm{m}^{3}$. Abaixo dessa classe, o sistema cut to length apresentou maior rendimento e, acima dela, o sistema full tree apresentou maior produtividade. Considera-se, então, que o sistema cut to length apresenta maior produtividade no processamento das árvores com VMls baixos (abaixo da classe 0,21 a $0,25 \mathrm{~m}^{3}$ ), enquanto o sistema full tree demonstra maior produtividade no processamento das árvores com VMIs altos (acima da classe 0,21 a 0,25 $\mathrm{m}^{3}$ ). Entretanto, Rosa e Oliveira (2014) ressaltam que os dados devem ser utilizados com prudência, visto que há possibilidade de alteração nos resultados devido aos defeitos na madeira, tais como galhos muito grossos, bifurcações e tortuosidades, além de problemas de ordem mecânica e operacional.

De acordo com a produção dos dois sistemas de colheita mecanizada, realizou-se a distribuição do volume em relação às classes de VMI produzidas, para análise da otimização entre os sistemas no período de 12 meses, observada na Figura 3. A classe de VMI 0,26 a 0,30 $\mathrm{m}^{3}$ foi a que mais produziu madeira processada, com $22,75 \%$ do total de produção no ano.

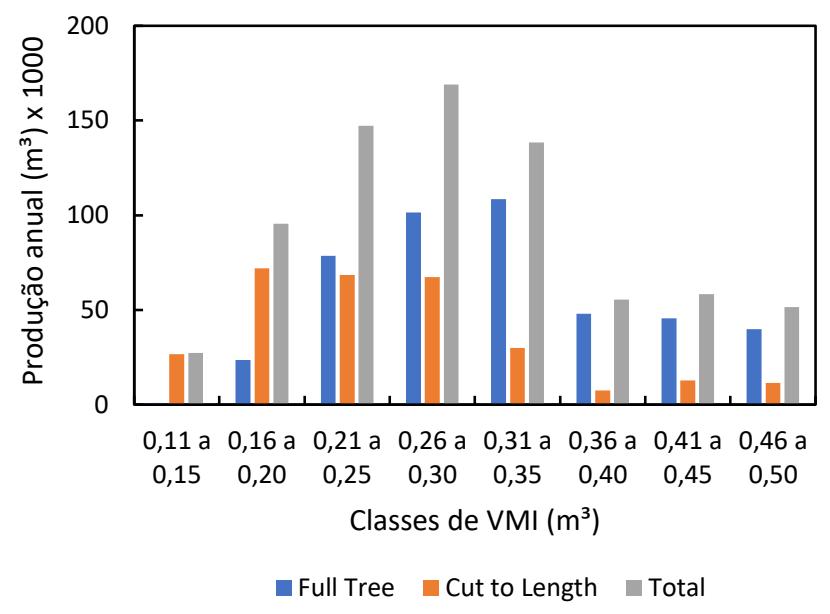

Figura 3. Produção anual e sua distribuição por classe de VMI e por sistema de colheita.

Como pode ser observado, na classe de VMI 0,16 a 0,20 $\mathrm{m}^{3}$, o maior processamento ocorreu com o uso do sistema full tree. De acordo com os resultados dos rendimentos, esse sistema é recomendado para o processamento de árvores com VMI acima da classe 0,21 a 0,25 $\mathrm{m}^{3}$. Da mesma forma, observou-se que o sistema cut to length processou muitas árvores na classe de VMI 0,26 a 0,30, acima do recomendado.

Em ambas as situações, pode haver ganho de produtividade se houver a substituição um sistema pelo outro, visto que o full tree é recomendado para árvores de maior VMI, ao passo que o cut to length é indicado para árvores de menor VMI. A readequação dos sistemas é importante, pois, segundo Oliveira Júnior et al. (2009), o elevado custo dos equipamentos de colheita florestal demanda seu uso pelo maior tempo possível com elevada produtividade.

Entretanto, ao se estudar a produtividade, deve-se considerar o sistema como um todo e não apenas as máquinas isoladamente (OLIVEIRA; PEREIRA, 2013). Como sistema, entenda-se os elementos e os processos relacionados com a cadeia de produção, bem como todas as atividades parciais, desde a derrubada da árvore até a madeira colocada no pátio da indústria (MALINOVSKI et al., 2008).

Cabe ainda ressaltar que este estudo foi realizado com equipamentos semelhantes e em condições operacionais semelhantes. Lopes et al. (2007), por exemplo, encontraram produtividade de $32,73 \mathrm{~m}^{3} \mathrm{~h}^{-1}$ em um 
harvester de esteiras ao deste estudo trabalhando em povoamentos de pinus com $0,47 \mathrm{~m}^{3}$ de $\mathrm{VMI}$, mas outro modelo de cabeçote. Ou seja, antes de afirmar qual sistema de colheita é o melhor, deve-se considerar todas as condições operacionais para só então realizar a análise dos rendimentos.

É com o olhar sobre a otimização das operações que devem ser realizados os estudos sobre as reais capacidades produtivas e as possíveis variáveis que interferem no rendimento, com vistas ao desenvolvimento de técnicas que melhorem o desempenho operacional (SILVA et al., 2003).

Este trabalho evidenciou que, mesmo com os sistemas de colheita da madeira trabalhando de forma independente e com resultados satisfatórios, pode-se otimizar as operações ao se direcionar os esforços de determinado sistema às características peculiares de cada situação operacional, como o VMI.

\section{CONCLUSÕES}

Há equilíbrio entre as produtividades do processamento em ambos os sistemas na classe de VMI 0,21 a $0,25 \mathrm{~m}^{3}$.

O sistema cut to length demonstra maior produtividade do processamento nas classes de VMI menores, enquanto o sistema full tree é superior nas classes de maior VMI, considerando a classe de equilíbrio.

\section{REFERÊNCIAS}

DRINKO, C. H.; LOPES, E. S.; OLIVEIRA, F. M. Produtividade e custos do corte de pinus com harvester de pneus e esteiras. Enciclopédia Biosfera, v. 11, n. 22, p. 3664-3677, 2015.

LOPES, E. S. Aplicação do programa SNAP III (Schedulingand Network AnalysisProgram) no planejamento da colheita e do transporte florestal. 2001. 150 f. Tese (Doutorado em Ciência Florestal) - Universidade Federal de Viçosa, Viçosa, 2011.

LOPES, E. S.; CRUZIANINI, E.; DIAS, A. N.; FIEDLER, N. C. Avaliação técnica e econômica do corte de madeira de pinus com cabeçote "harvester" em diferentes condições operacionais. Floresta, v. 37, n. 3, p. 305-13, 2007.

MACHADO, C. C. Colheita Florestal. 3. ed. Viçosa: Editora UFV, 2014. 543 p.

MALINOVSKI J. R., CAMARGO, S. M. C., MALINOVSKI, A. R., MALINOVSKI A. R. Sistemas. In: MACHADO, C. C. Colheita Florestal. 3. ed. Viçosa: Editora UFV. 2014, p. 162-184.

OLIVEIRA JÚNIOR, E. D.; SEIXAS, F.; BATISTA, J. L. F. Produtividade de feller buncher em povoamento de eucalipto em relevo acidentado. Floresta, v. 39, n. 4, p. 905-912, 2009.
OLIVEIRA, F. M.; PEREIRA, A. L. S. Comparação da produtividade entre equipamentos em dois sistemas de colheita de madeira de eucalipto. Revista Científica Eletrônica de Engenharia Florestal, v. 22, n. 1, p. 30-40, 2013.

ROSA, M. O.; OLIVEIRA, F. M. Análise da qualidade do processamento de madeira em dois sistemas mecanizados de colheita florestal. Revista da União Latino-americana de Tecnologia, n. 2, p. 19-37, 2014.

SILVA, C. B.; SANT'ANNA, C. M.; MINETTE, L. J. Avaliação ergonômica do feller-buncher utilizado na colheita de eucalipto. Cerne, v. 9, n. 1, p. 109-118, 2003. 